\title{
The LIM protein Ajuba promotes adipogenesis by enhancing PPARY and P300/CBP interaction
}

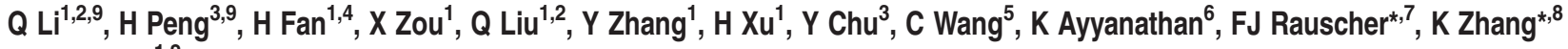 \\ and $\mathrm{Z} \mathrm{Hou}^{\star, 1,2}$
}

Adipocytes play a vital role in energy homeostasis and adipogenesis is a hierarchically regulated cellular differentiation process, in which the precursor mesenchymal stem cells are differentiated into mature adipocytes. Here, we report Ajuba is an important regulator of adipocyte differentiation by functioning as an obligate co-activator of PPAR $\gamma$. Ajuba binds the DNA-binding domain of PPAR $\gamma$ via its preLIM region in a ligand-independent manner. Depletion of Ajuba in 3T3-L1 cells decreases PPAR $\gamma$ target gene expression and results in delayed adipogenic differentiation. Conversely, stable overexpression of Ajuba in 3T3-L1 cells increases PPAR $\gamma$ target gene expression and accelerates adipogenic differentiation. Mechanistic investigations demonstrate that Ajuba recruits p300/CBP via its LIM domain and facilitates p300/CBP binding to PPAR $\gamma$. Moreover, Ajuba, PPAR $\gamma$, p300/CBP can cooperatively occupy the PPAR $\gamma$ target promoters and concomitantly increases histone acetylation at these loci. Collectively, these data suggest that Ajuba is a co-activator constitutively associated with PPAR $\gamma$ and may be a potential therapeutic target for PPAR $\gamma$ mediated metabolic disorders.

Cell Death and Differentiation (2016) 23, 158-168; doi:10.1038/cdd.2015.83; published online 26 June 2015

Adipocyte differentiation is a process of multipotent mesenchymal stem cells (MSCs) undergoing commitment to become preadipocytes, which further differentiate into mature adipocytes. This process is hierarchically regulated by layers of signaling pathways. BMPs, Wnt, Hedgehog and Retinoblastoma protein regulate the conversion of MSCs to preadipocytes, whereas combining signaling pathways stimulated by insulin, glucocorticoid, cAMP and their downstream transcription factor cascades, such as C/EBPs (CCAAT/ enhancer-binding proteins), and PPARy are precisely orchestrated to control differentiation of preadipocytes into mature adipocytes. $^{1-4}$

PPARy is a member of the nuclear receptor superfamily of ligand-dependent transcription factors. PPARy is highly expressed in adipose tissue and functions as a master regulator of the adipocyte differentiation. ${ }^{5,6}$ This is firmly supported by the PPARy-null mice that are completely devoid of adipose tissue. ${ }^{7}$ As a result of utilizing different promoter and alternative splicing, PPARy gene produces two protein isoforms, named PPARy1 and PPARy2, with the latter containing additional 30 amino acids at the extreme $\mathrm{N}$-terminus. For the more specific distribution in adipose tissue than PPARy1, PPARy2 is thought possessing unique role in adipogenesis. ${ }^{6,8}$ PPARy forms a heterodimer with RXR that recognizes the PPARy response element PPRE (DR1, PuCCT/ACA) to control its target gene transcription. Like other nuclear receptors, PPARy contains four domains from the $\mathrm{N}$-terminus to $\mathrm{C}$-terminus: $\mathrm{A} / \mathrm{B}$ domain (AF1 domain), $\mathrm{C}$ domain (DNA-binding domain), D domain (Hinge domain), E/F domain (AF2 domain/ligand-binding domain). ${ }^{5,6}$ The A/B domain possesses ligand-independent transactivity by directly recruiting co-activators, such as PGC2 and histone acetyltransferase p300/CBP. ${ }^{9,10}$ The DNA-binding domain encompasses two C4-type zinc-fingers mediating the interaction with PPRE. The $D$ domain is the hinge connecting $C$ and $E / F$ domains. There are also co-regulators binding to the $\mathrm{C}$ or D domain, like PGC-1a, which functions as a key coregulator of PPARy in the adipocytes browning. ${ }^{11,12}$ The AF2 domain mediates the ligand binding, most co-regulators docking and the heterodimerization. Co-regulators bind this site through their conservative NR boxes (LXXLL), and this binding is strictly regulated by ligand: in the absence of ligand, co-repressors like NcoR/SMRT are docked at this domain, whereas in the presence of ligand, those co-repressors are replaced by co-activators such as SRC and p300/CBP. ${ }^{5,6,13}$

Ajuba is a member of the LIM protein family characterized by containing tandem LIM domains at the $\mathrm{C}$ termini. The LIM domain is a double zinc-fingers in structure and was initially

\footnotetext{
${ }^{1}$ Hongqiao Institute of Medicine, Shanghai Tongren Hospital/Faculty of Basic Medicine, Department of Biochemistry and Molecular Cell Biology/Shanghai Key Laboratory for Tumor Microenvironment and Inflammation, Shanghai Jiaotong University School of Medicine, Shanghai, China; ${ }^{2}$ Institute of Cancer Stem cells, Dalian Medical University Cancer Center, Liaoning, China; ${ }^{3}$ Digestive Endoscopy Center, Shanghai Tongren Hospital, Shanghai Jiaotong University School of Medicine, Shanghai, China; ${ }^{4}$ Department of Endocrinology, Renji Hospital, Shanghai Jiaotong University School of Medicine, Shanghai, China; Institute of Biomedical Sciences, East China Normal University, Shanghai, 200241, China; ${ }^{6}$ Department of Biological Sciences, Florida Atlantic University, 777 Glades Road, Boca Raton 33431, FL, USA; ${ }^{7}$ Wistar Institute, 3601 Spruce Street, Philadelphia, 19104, PA, USA and ${ }^{8}$ Department of Cell Biology, Dalian Medical University, Liaoning, China

*Corresponding author: FJ Rauscher, Wistar Institute, 3601 Spruce Street, Philadelphia, 19104, PA, USA. E-mail: rauscher@wistar.org

or K Zhang, 9 Western Section, Lvshun South Street, Dalian 116044, China. E-mail: 18940993315@163.com

or Z Hou, Hongqiao Institute of Medicine, Shanghai Tongren Hospital/Faculty of Basic Medicine, Department of Biochemistry and Molecular Cell Biology/Shanghai Key Laboratory for Tumor Microenvironment and Inflammation, Shanghai Jiaotong University School of Medicine, 280 South Chongqing Road, Building 7\#, Room 110, Shanghai 200025, China. Tel: +86 15121148204; Fax: +86 021 63858578; E-mail: houzy@ @jtu.edu.cn

${ }^{9}$ These authors contributed equally to this work.

Abbreviations: Rosi, rosiglitozone; PPAR $\gamma$, peroxisome proliferator-activated receptor gamma; Co-IP, co-immunoprecipitation; ChIP, chromatin immunoprecipitation Received 12.12.14; revised 18.5.15; accepted 20.5.15; Edited by JP Medema; published online 26.6.15
} 
identified in Caenorhabditis elegans Lin-11, rat Isl-1 and C. elegans Mec-3, from which the acronym LIM was derived. ${ }^{14,15}$ The LIM protein family can be subdivided into different subfamilies according to sequence homology within the LIM domains, the number of LIM domains and their organization within the proteins. Ajuba belongs to Zyxin/Ajuba subfamily, including Zyxin, Ajuba, Trip6, Wtip, Limd1 and Lpp. The Ajuba/ Zyxin subfamily has not yet been uncovered to bind the DNA elements, and their LIM domains mainly function as proteinprotein interface. ${ }^{16-18}$ An unique nucleus exporting signal is localized in the preLIM region of Ajuba (aa NO. 289-297) and deletion of the nucleus exporting signal or the whole preLIM region will result in the nucleus accumulation of Ajuba. ${ }^{16,19}$ In addition, the preLIM region also mediates the protein-protein interaction, such as HDACs and Prmt5. ${ }^{20,21}$ Depending on cell types, Ajuba can be located near the membrane, in the cytoplasm and/or in the nucleus and the stimulation of signals or interaction with proteins will also change its localization in cells. ${ }^{16-18,22}$ Ajuba functions as a scaffold involving assembly of multiple protein complexes to regulate cell adhesion, migration, mitosis, microRNA maturation, cell differentiation and tissue development. ${ }^{16-31}$

Ajuba can directly participate in transcriptional regulation where it serves as a transcriptional co-repressor and downregulate gene expression. We lately identified Ajuba as a co-repressor for Snail and as an essential regulator of mesenchymal-epithelial transition and metastasis. Ajuba further recruits Prmt5 and HDACs through its preLIM region to repress E-cadherin expression, a known Snail target gene. ${ }^{17,18,20,21}$ Moreover, we demonstrated that Ajuba contains functional NR boxes and directly interacts with RAR $a,{ }^{22}$ where Ajuba functions as a co-repressor and negatively regulates RAR signaling. Here, we describe the novel findings that Ajuba interacts with PPARy via its non-NR box module within the preLIM region and functions as a co-activator for PPARy by recruiting p300/CBP via its LIM domain.

\section{Results}

Ajuba is a novel PPAR $\boldsymbol{y}$-interacting protein. To examine if Ajuba physically interacts with PPAR $\gamma$, we transiently co-expressed Myc-Ajuba and Flag-PPARy1 or Flag-PPARy2 in $293 \mathrm{~T}$ cells and the co-immunoprecipitation (co-IP) assays were carried out using Flag antibody. The co-eluted proteins were detected by western blot assays. Indeed, both Flag-PPAR 1 and PPAR 2 robustly interacted with Ajuba (Figure 1a). Given the high similarity between PPARy1 and PPARY2, and the importance in the regulation of adipogenesis, we selected PPARy2 for our further investigation. a
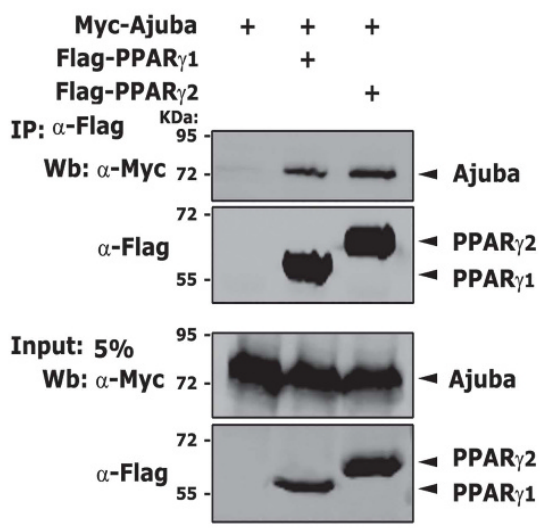

C

3T3-L1 cells Differentiation

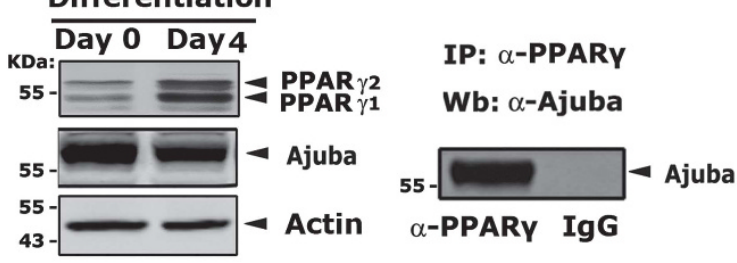

b

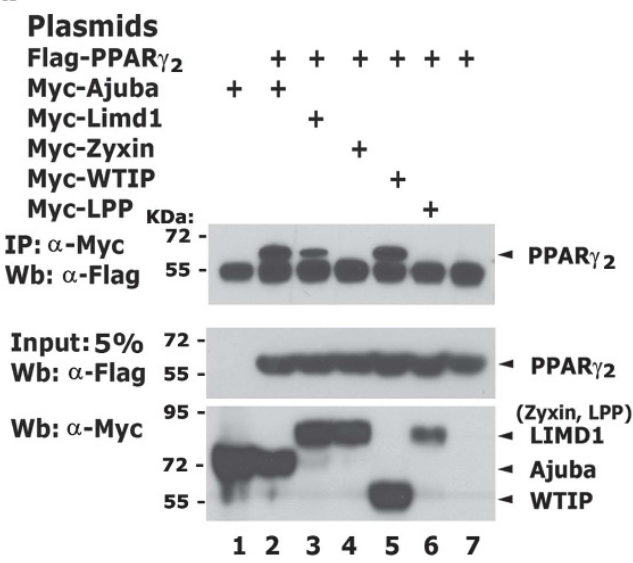

d

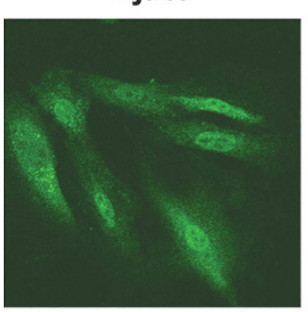

PPAR $\gamma$

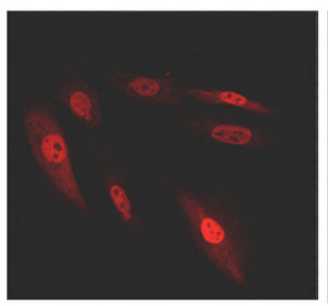

IF in 3T3-L1 cells

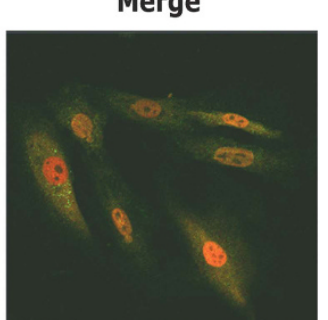

Cell lysates were prepared from Figure 1 Ajuba is a novel PPAR $\gamma$-interacting protein. (a) Ajuba binds both PPAR $\gamma 1$ and PPAR $\gamma 2$ when co-expressed in 293 T cells transiently co-expressing Myc-Ajuba and Flag-PPAR $\gamma 1$ or Flag-PPAR $\gamma 2$. The co-IP assays were carried out with Flag antibody and the co-eluted proteins were
293 . detected by western blot assays with Myc and Flag antibodies. (b) PPAR $\gamma$ selectively interacts with members of Ajuba family. Flag-PPARy2 and Myc-Ajuba, LimD1, WTIP, Zyxin, LPP were co-expressed in $293 \mathrm{~T}$ cells and co-IP assays were performed with Flag antibody. The co-eluted proteins were detected by western blot assays with Myc antibody. ${ }^{*}$ Non-specific binding. (c) The endogenous interaction of Ajuba and PPAR in 3T3-L1 cells. Whole-cell lysates were prepared from 3T3-L1 cells undergone differentiation program at day 4 and were incubated with antibody specific to PPAR $\gamma$. The co-eluted proteins were detected by using Ajuba antibody. (d) Indirect IF assays show the subcellular localization of Ajuba and PPAR $\gamma$ in 3T3-L1 cells. 3T3-L1 cells at normal culture condition were prepared for IF staining assays using Ajuba and PPAR $\gamma$ antibodies, and the images were taken by confocal microscopy 
To examine the interaction between PPARy and other members of Ajuba/Zyxin family, we co-expressed PPARy2 and Ajuba, LimD1, Wtip, Zyxin or Lpp in 293 T cells and performed co-IP assays. In addition to Ajuba, LimD1 and WTIP also interacted with PPARY2 (Figure 1b, lanes 2, 3, 5), whereas Zyxin and Lpp did not (Figure 1b, lanes 4, 6), suggesting that PPARy selectively interacts with members of Ajuba/Zyxin family.

To further confirm the interaction of the endogenous Ajuba and PPAR $y$, we first performed western blot assays to examine the expression of PPARy and Ajuba in 3T3-L1 cells before and after the adipogenic induction. Both PPARy1 and PPARy2 were readily detected in 3T3-L1 cells, but were markedly induced after 4 days adipogenic induction (Figure 1c, left panel). Whole-cell lysates prepared from 3T3-L1 cells stimulated with the induction cocktail for 4 days were incubated with antibody specifically recognizing both PPAR 1 and PPARY2, and the co-eluted Ajuba proteins were robustly detected, indicating that PPARy interacted with Ajuba at the endogenous level (Figure 1c, right panel). Next, we performed indirect immunofluorescence (IF) staining assays to examine the subcellular localization of the Ajuba and PPAR $y$ proteins in 3T3-L1 cells, and the Ajuba and PPARy proteins were visualized by confocal microscopy. We observed that Ajuba could be found in the cytoplasm and nucleus, whereas PPARy was predominately in the nucleus (Figure 1d), in which the distribution patterns of Ajuba and PPAR $y$ were overlapped. Collectively, these data clearly demonstrate that Ajuba is a novel PPARy-interacting protein.

The preLIM region of Ajuba directly binds the DNAbinding domain of PPAR $\boldsymbol{y}$. To identify the regions that mediate the interaction between Ajuba and PPARy, we first made series of Myc-epitope-tagged truncation mutants of Ajuba (Figure 2a). We co-expressed full-length Flag-PPARy2 and Ajuba or its truncation mutants in $293 \mathrm{~T}$ cells, and co-IP assays were performed with Flag antibody. Notably, the LIM domain alone did not bind PPARy2 (Figure 2b, lane 15), whereas the preLIM-347 and preLIM-312 displayed similar binding affinity to PPARY2 as that of the full-length Ajuba did (Figure 2b, lanes 11-13); the preLIM-279 failed to bind PPARy2 (Figure 2b, lane 14), suggesting that the amino-acid residues between No. 279 and No. 312 are critical for PPARy binding.

To determine the regions in PPAR $y$ involved in Ajuba binding, we made truncation mutants of Flag-PPARy2, which were co-expressed with Myc-Ajuba in 293 T cells (Figure 2c). Co-IP assays were carried out with Flag antibody and the coeluted proteins were detected with Myc antibody. The PPARy2 mutants Flag- $\Delta \mathrm{A} / \mathrm{B}$, Flag- $\Delta \mathrm{E} / \mathrm{F}$, Flag- $\Delta \mathrm{A} / \mathrm{B} / \mathrm{E} / \mathrm{F}$ and Flag- $\Delta \mathrm{D}$ (Figure 2d, lanes $3,4,5,8$ ) displayed similar binding affinity to Ajuba as that of the full-length PPARy2 did (Figure 2d, lane 2), whereas Flag- $\Delta \mathrm{C} / \mathrm{D}$ or Flag- $\Delta \mathrm{C}$ showed weak or none binding (Figure $2 \mathrm{~d}$, lanes 6,7 ), indicating that the $C$ domain is the predominate binding region for Ajuba.

To further determine whether Ajuba directly binds the $C$ domain of PPAR $y$, we expressed GST- PPARy-C and His-Ajuba proteins from Escherichia coli, respectively, and these proteins were purified for in vitro binding assays. The co-IP assays were performed using GST antibody and the co-eluted His-Ajuba was detected by Ajuba antibody. Indeed, GST- PPARy-C binds His-Ajuba in vitro (Figure $2 \mathrm{e}$ ), indicating Ajuba directly interacts with the $\mathrm{C}$ domain of PPAR $y$.

To confirm if the binding of Ajuba to PPARy is regulated by ligand, we co-expressed Myc-Ajuba and Flag-PPARy2 in 293 T cells and the cells were treated with various doses of Rosi for $12 \mathrm{~h}$ before harvesting. Co-IP assays indicated that the interaction between Ajuba and PPARy2 was not apparently affected by ligand treatment (Figure 2f). As a positive control, Rosi increased the binding between PPARy2 and CBP (Figure 2g). To further confirm the observation at the endogenous level, whole-cell extracts prepared from 3T3-L1 cells undergone differentiation program (without Rosi) for 4 days and then treated with DMSO or Rosi for $12 \mathrm{~h}$ before harvesting. Co-IP assays were performed with antibody specific to PPARy and the co-eluted proteins were detected by western blot assays with Ajuba and CBP antibody. Consistently, Rosi stimulation did not affect the binding between Ajuba and PPAR $\gamma$, but markedly increased CBP binding to PPARy (Supplementary Figure 1). Taken together, these results demonstrate that Ajuba binds to the DNA-binding domain of PPARy in a ligand-independent manner.

Ajuba is required for PPAR $\boldsymbol{y}$ to maximally transactivate target gene expression. To examine the role of Ajuba in PPARy-mediated transcriptional activity, we first depleted Ajuba in 3T3-L1 cells using lentiviral shRNA specifically targeting Ajuba (Figure 3a). ${ }^{22}$ The resulting cells were then treated with Rosi $(1 \mu \mathrm{M})$ or DMSO for $16 \mathrm{~h}$ and the total mRNAs were extracted for qRT-PCR analysis. We chose FABP4, LPL, PLIN-1 and CD36, the known target genes of PPAR $\gamma$, as representatives to evaluate the transcriptional activity of PPARy. ${ }^{32-35}$ Surprisingly, depletion of Ajuba in 3T3-L1 cells resulted in significantly decreased expression of these target genes at normal culture media (DMSO) or Rosi treatment (Figure $3 b-e)$. Conversely, stable overexpression of Ajuba resulted in elevated expression of these target genes (Figure $3 \mathrm{~g}$ and $\mathrm{h}$ ). Notably, depletion or forced expression of Ajuba in 3T3-L1 cells did not apparently affect the expression of PPARy (Figure $3 f$ and $h$ ), indicating that altered PPAR $y$ target gene expression by Ajuba is not due to the fluctuated expression of PPAR $y$. Taken together, these data demonstrate that Ajuba is an important activator for PPAR $y$-mediated transcriptional activity.

Ajuba and PPARy cooperatively bind to PPAR $\boldsymbol{y}$ target promoters. To determine whether Ajuba directly modulates PPAR $y$ target genes, we cloned mouse LPL gene promoter containing a functional PPRE (-130-118) into pGL3 basic luciferase vector to make a LPL-Luc reporter (Figure 4a). LPL-Luc reporter, together with plasmids encoding FlagPPARy2 and Myc-Ajuba, was transiently transfected into $293 \mathrm{~T}$ cells and the luciferase activity was normalized to $\beta$-galatosidase activity. Ajuba or PPAR 2 alone weakly or modestly increased LPL-Luc activity, whereas co-expression of Ajuba and PPARY2 markedly induced LPL-Luc activity (Figure 4b), suggesting a synergy may exist between Ajuba and PPARy to transactivate target gene expression.

Next, we performed chromatin immunoprecipatation (ChIP) assays in $293 \mathrm{~T}$ cells transiently expressing Flag-PPARy2 or 
a

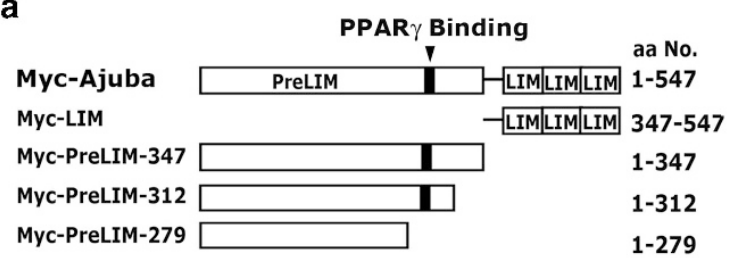

b
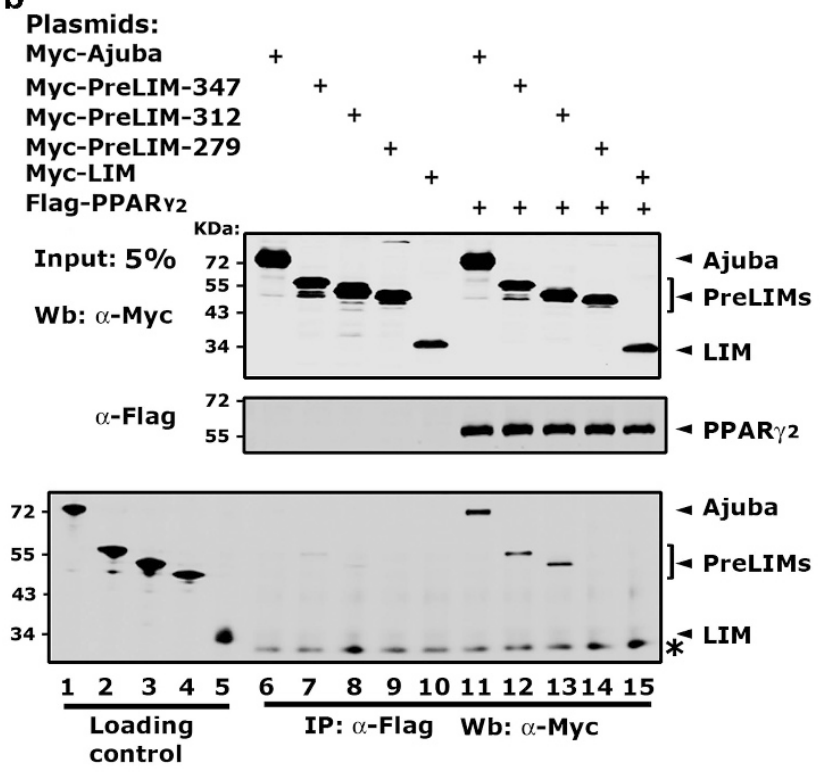

C

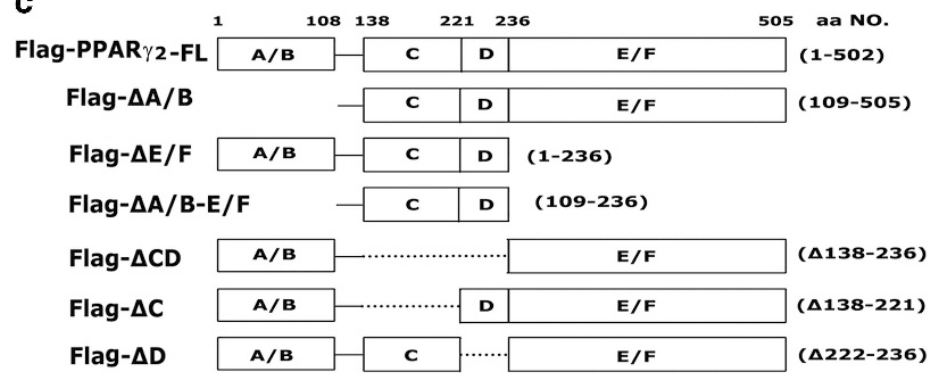

e

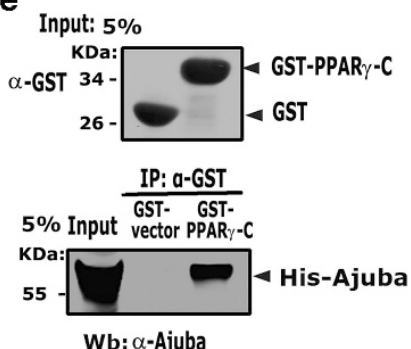

f

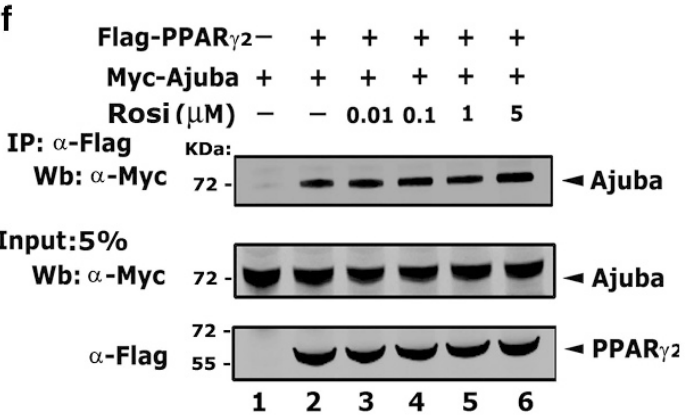

d

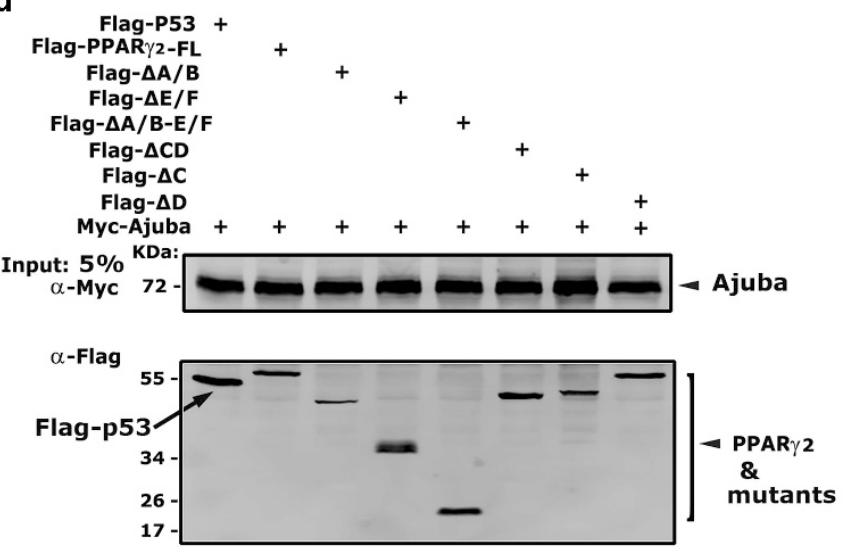

IP: $\alpha$-Flag

Wb: $\alpha-$ Myc

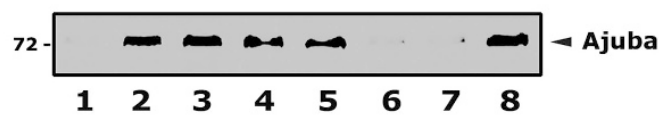

g

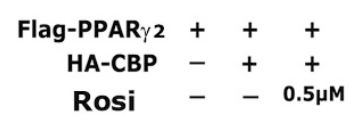

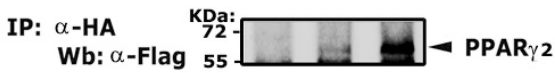

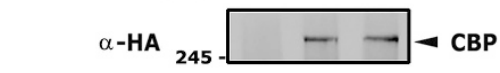

Input: $5 \%$
Wb: $\alpha-$ Flag
$55-$

Figure 2 The preLIM region of Ajuba directly binds the DNA-binding domain (c domain) of PPAR $\gamma$. (a) A diagram shows series of Myc-epitope-tagged truncation mutants of Ajuba. (b) The amino-acid residues between No. 279 and No. 312 are critical for PPAR $\gamma$ binding. Flag-PPAR 2 and Myc-Ajuba or its truncation mutants were co-expressed in $293 \mathrm{~T}$ cells and co-IP assays was performed with Flag antibody. The co-eluted proteins were detected by western blot assay with Myc antibody. (c) A diagram shows series of Flag-epitope-tagged truncation mutants of PPAR $\gamma 2$. (d) The $\mathrm{C}$ domain of PPAR $\gamma$ is required for Ajuba binding. Flag-PPAR 2 and truncation mutants were co-expressed with Myc-Ajuba in $293 \mathrm{~T}$ cells. Co-IP assays were carried out with Flag antibody and the co-eluted proteins were detected with Myc antibody. Flag-P53 served as a negative control. (e) In vitro binding of the full-length Ajuba and the $\mathrm{C}$ domain of PPAR $\gamma$. GST-PPAR $\gamma-\mathrm{C}$ and His-Ajuba proteins were, respectively, expressed in E. coli, and were purified using GST- or Ni-beads. The in vitro binding assays were performed in buffer containing $20 \mathrm{mM}$ Tris (pH 7.5), $150 \mathrm{mM} \mathrm{NaCl}, 2.5 \mathrm{mM}$ EDTA and 0.5\% NP-40. The co-IP assays were performed using GST beads, and co-eluted His-Ajuba was detected by Ajuba antibody. (f) Ajuba binds to PPAR $\gamma$ in a ligand-independent manner. Myc-Ajuba and Flag-PPAR 2 were co-expressed in $293 \mathrm{~T}$ cells and the cells were treated with various doses of Rosiglitazone (Rosi) for $12 \mathrm{~h}$ before harvesting. The co-IP assays were carried out with Flag antibody. (g) As a positive control, Rosi treatment increases the binding between PPAR $\gamma 2$ and CBP

Myc-Ajuba or in combination, together with the LPL-Luc reporter by using Flag or Myc antibodies (Figure 4c), and the co-eluted LPL-Luc reporter DNA fragments were examined by quantitative PCR (qPCR) analyses. Expression of PPAR 2 alone resulted in enrichment of the LPL promoter DNA to 7\% relative to the input control (the non-expression of PPARy2, Lane 1, was designated as $1 \%$ ), but when co-expressed with Ajuba, the enriched LPL promoter DNA was increased to $18 \%$ (Figure 4d, left panel). Similarly, expression of Ajuba alone resulted in slight enrichment of LPL promoter DNA, whereas 
a
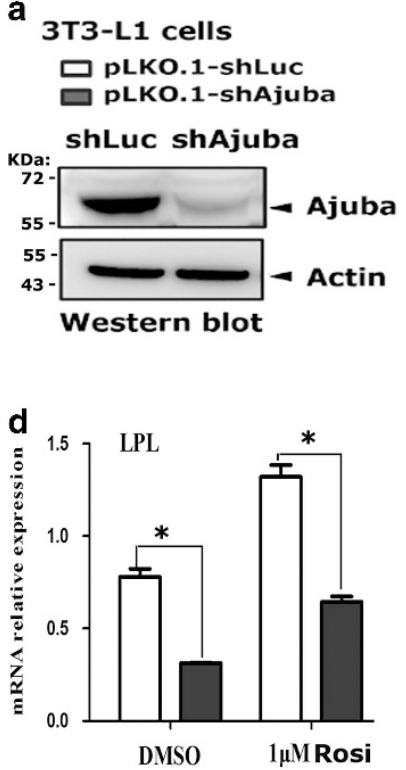

g

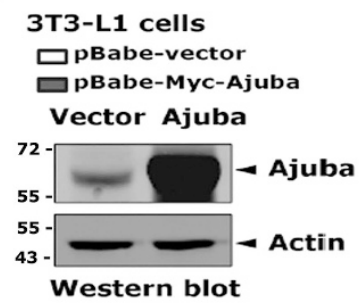

b

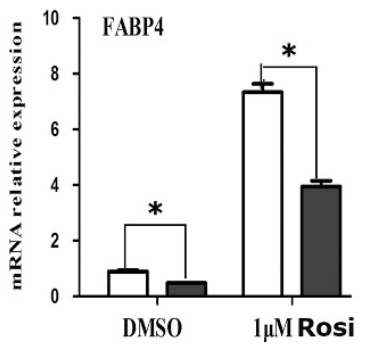

e

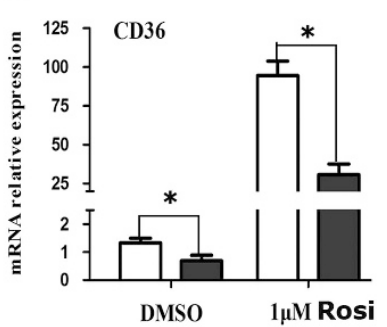

C

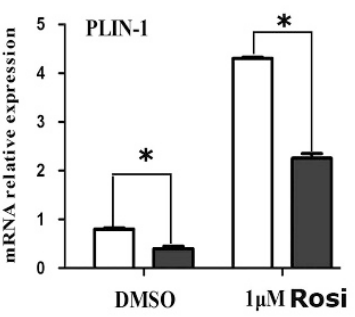

f
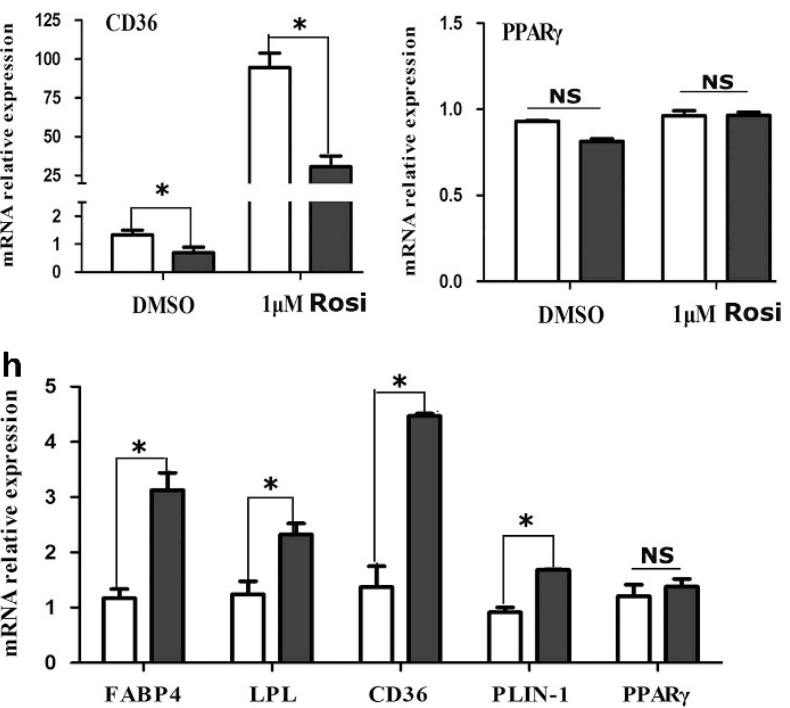

Figure 3 Ajuba is required for PPAR $\gamma$ to maximally transactivate target gene expression. (a) Ajuba was effectively depleted in 3T3-L1 cells using specific targeting lentiviral shRNA. The shAjuba vector was used previously to deplete Ajuba ${ }^{22}$ and was introduced into 3 T3-L1 cells via viral infection. Pooled stable cells were established after puromycin selection and were analyzed by western blots. (b-f) The deletion of Ajuba decreases PPAR $\gamma$ target genes expression. The qRT-PCR analysis of FABP4, PLN-1, LPL, CD36 and PPAR $\gamma$ expression in 3T3-L1-shLuc and 3T3-L1-shAjuba cells treated with Rosi (1 $\mu \mathrm{M})$ or DMSO for $16 \mathrm{~h}$. (g) Stable expression of Ajuba in 3T3-L1 cells via viral infection. (h) The overexpression of Ajuba increases PPAR $\gamma$ target genes expression. Expression of FABP4, PLIN-1, LPL, CD36 and PPAR $\gamma$ were measured by qRT-PCR assays. Data were shown as mean \pm S.D. from three independent experiments, ${ }^{*} P<0.05$

co-expression of PPARy2 and Ajuba greatly increased the binding of Ajuba to the LPL promoter (Figure 4d, right panel). These data indicate that the binding of Ajuba to LPL promoter is largely dependent on the presence of PPAR $y$, and in turn Ajuba can enhance the DNA binding of PPAR $y$.

To further examine the binding activity of PPAR $y$ and Ajuba at the endogenous target chromatins, we performed ChIP assays in 3T3-L1 cells at normal culture condition or at a condition stimulated with the adipogenic induction cocktail for 4 days to stimulate PPARy expression (Figure 1c). Antibodies specific to PPARy and Ajuba were used to perform ChIP assays and the co-eluted DNA fragments were amplified using primer sets flanking the functional PPREs of the promoters of CD36 and LPL genes, respectively. In 3T3-L1 cells at preadipocyte stage, both PPARy and Ajuba weakly bound the PPRE loci of CD36 and LPL genes, but when PPARy was robustly induced after 4 days adipogenic induction, PPARy binding at the target promoters was drastically increased, and concomitantly Ajuba binding was markedly increased at the same loci (Figure 4e). Taken together, these results indicate that Ajuba cooperates with PPAR $y$ at the target chromatins to activate gene transcription.
Ajuba is an important modulator for adipogenic differentiation in 3T3-L1 cells. To examine if Ajuba participates in the PPAR $\gamma$-dependent adipogenesis, 3T3-L1-shLuc and 3T3-L1-shAjuba cells were subjected to the adipogenic induction. At days 4 after induction, small fat droplets began to appear and at days 8 the majority of the cells were differentiated into adipocytes containing large fat droplets (Figures 5a and c). Strikingly, in 3T3-L1-shAjuba cells, the differentiation process was apparently inhibited, reflected by fewer cells containing fat droplets even at days 8 (Figure 5a). We also quantified the differentiation degree by measuring the red-staining intensity (Figure 5a). Consistently, expression of the adipocyte marker genes, which are also PPARy well-known target genes, including FABP4, LPL, CD36, GLUT4, C/EBPa and PLIN-1, was much lower in 3T3-L1-shAjuba cells at days 6 and 8 compared with that in 3T3-L1-shLuc cells (Figure 5b). Conversely, stable overexpression of Ajuba in 3T3-L1 cells evidently accelerated adipocyte maturation reflected by earlier and more fat droplet formation and the higher expression level of adipocyte marker genes in 3T3-L1Ajuba cells (Figures $5 \mathrm{c}$ and d). Together, these observations 
a

PGL3.0-LPL-LUC

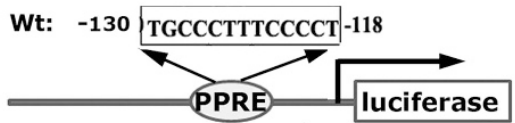

b

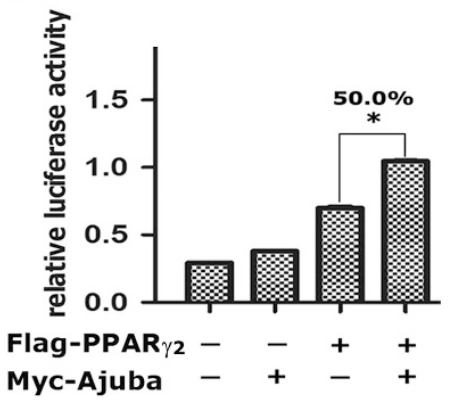

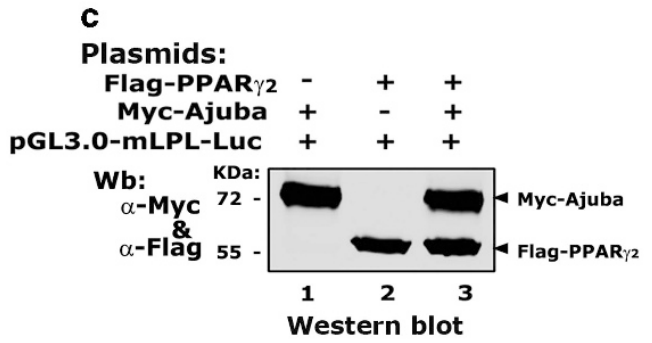

e

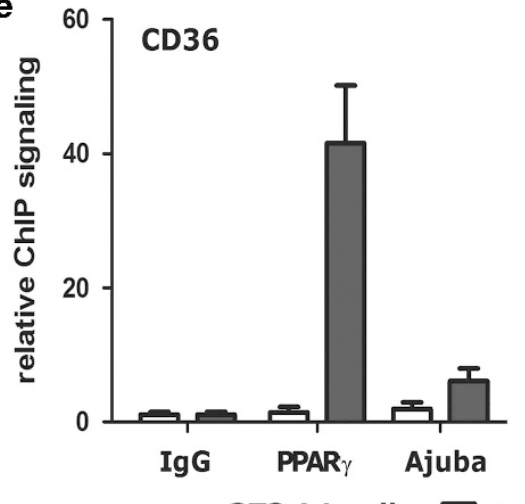

d

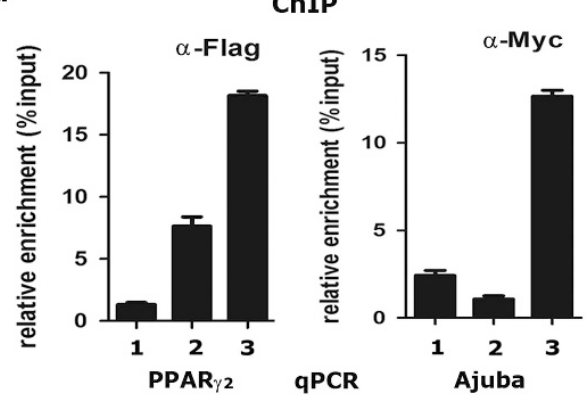

Figure 4 Ajuba and PPAR $\gamma$ cooperatively bind to PPAR $\gamma$ target promoters. (a) Diagram shows the mouse LPL promoter. Mouse LPL gene promoter containing a functional PPRE (-130- 118) was cloned into pGL3 basic luciferase vector to create a LPL-Luc reporter. (b) Co-expression of Ajuba and PPARy2 maximally induced LPL-Luc activity. LPL-Luc reporters, together with plasmids encoding Flag-PPAR $\gamma 2$ and Myc-Ajuba were transfected into $293 \mathrm{~T}$ cells and LPL-Luc reporter activity was assayed. The value was normalized to $\beta$-gal activity. Data were shown as mean \pm S.D. from three independent experiments, ${ }^{\star} P<0.05$. (c) Western blots show the expression of PPAR 2 and Ajuba proteins transiently transfected into $293 \mathrm{~T}$ cells. (d) ChIP assays show binding activity of Ajuba and PPAR 2 to LPL promoter in $293 \mathrm{~T}$ cells. ChIP assays were performed using Flag (mouse) and Myc (mouse) antibodies, and the co-eluted DNA fragments were analyzed by using qPCR. (e) ChIP assays in 3T3-L1 cells at normal culture (preadipocytes) or in differentiated 3T3-L1 cells at day 4 (adipocytes) were performed using antibodies specific to PPAR $\gamma$ and Ajuba. The co-eluted DNA fragments were amplified using primer sets flanking the functional PPREs at the promoters of CD36 or LPL, respectively, and were normalized to the inputs. Data: mean \pm S.D. from three independent experiments

clearly demonstrate that Ajuba has an important role in the adipogenesis of 3T3-L1 cells.

Ajuba interacts with p300/CBP. p300/CBP are the predominate enzymes recruited to PPARy to transactivate gene expression, thus we speculated that Ajuba may also recruit p300/CBP to PPARy. Indeed, Ajuba interacted with both p300 and CBP when co-expressed in $293 \mathrm{~T}$ cells (Figures 6a and b). We further observed that endogenous Ajuba could also immunoprecipitated the endogenous p300 and CBP in 3T3-L1 cells (Figure 6c).
To identify regions in Ajuba responsible for the interaction with $\mathrm{p} 300 / \mathrm{CBP}$, we co-expressed HA-CBP and full-length Myc-Ajuba, Myc-preLIM-347 or Myc-LIM in 293 T cells and co-IP assays were performed with Myc antibody. Western blot assays showed that the full-length Ajuba and the truncated LIM domain bound HA-CBP, whereas the preLIM-347 domain failed to bind CBP (Figure $6 \mathrm{~d}$ ). Collectively, these data suggest that Ajuba may recruit p300/CBP to PPARy via its LIM domain.

Ajuba recruits p300/CBP to PPAR $\boldsymbol{y}$. To determine whether PPAR $y$, Ajuba and p300/CBP form functional ternary complexes, we co-expressed Flag-PPARy2 and HA-CBP, together 
a
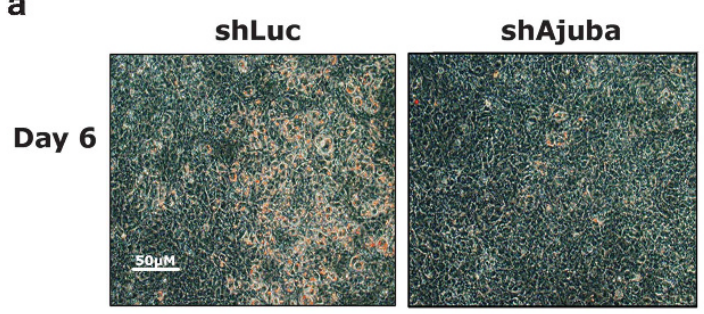

Day 8
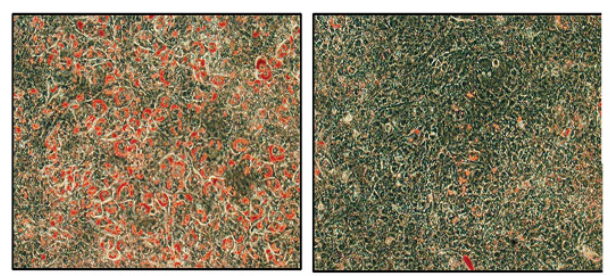

3T3-L1 cells (Oil red 0 staining)

C

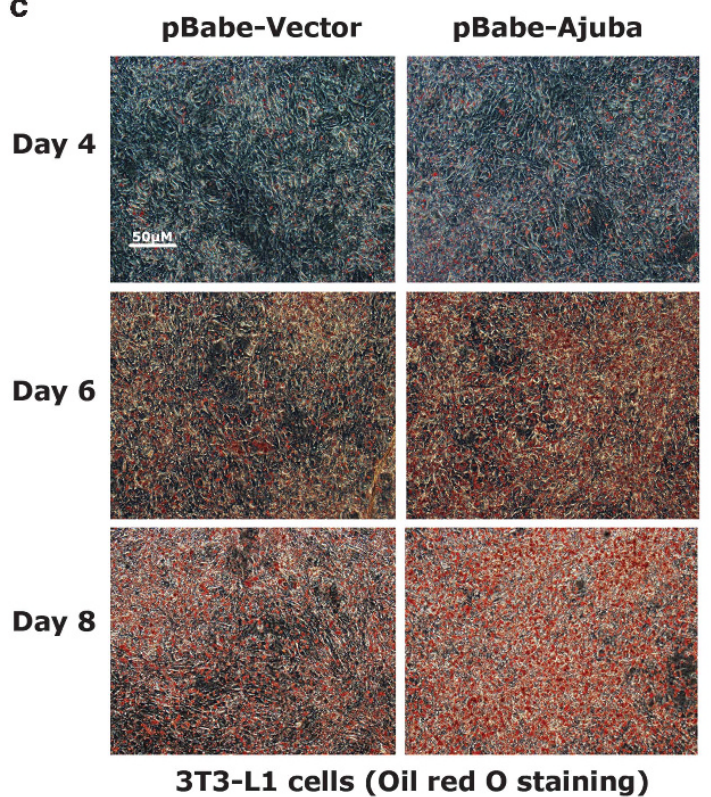

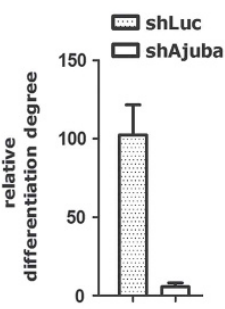
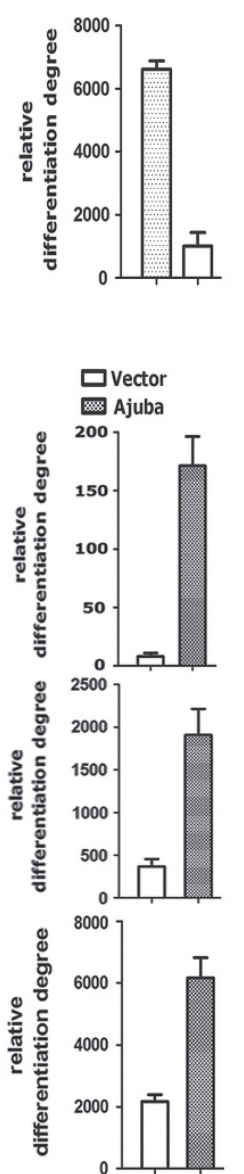

b

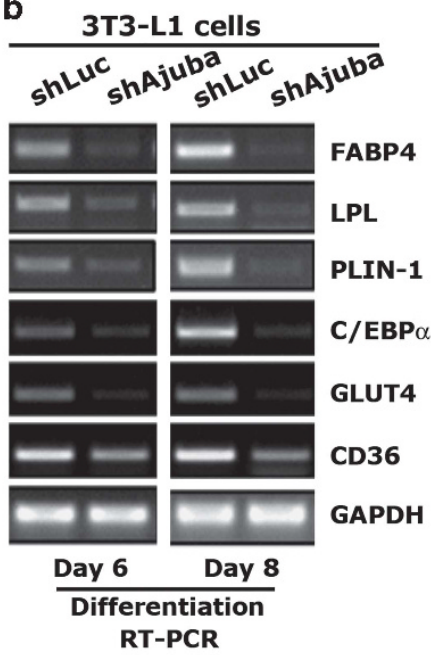

d

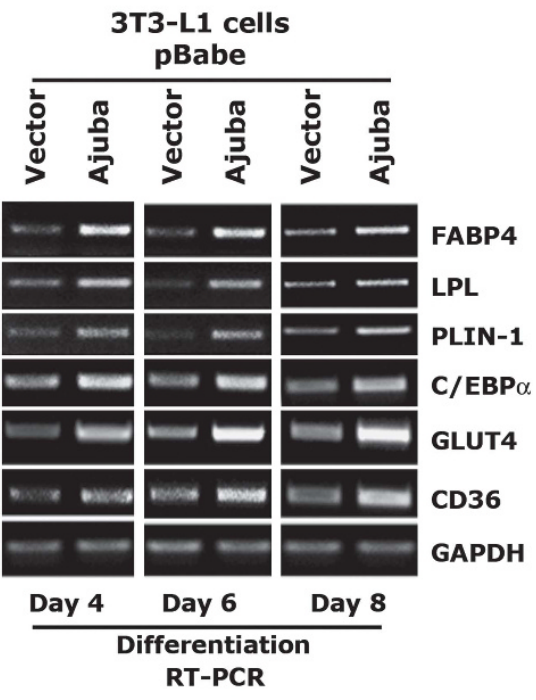

Figure 5 Ajuba is an important modulator for adipogenic differentiation in 3T3-L1 cells. (a) Depletion of Ajuba inhibits adipocyte differentiation in 3T3-L1 cells. 3T3-L1-shLuc and 3T3-L1-shAjuba cells were subjected to the adipogenenic induction. The day adding the cocktail (0.5 mM IBMX, $1 \mu$ M Dexamethasone, $10 \mu \mathrm{g} / \mathrm{ml}$ Insulin and $2.5 \mu \mathrm{M}$ Rosi) was designated as day 0 . Adipocytes were visualized by method of the Oil-Red-O Staining. The differentiation degree was quantified by averaging red-staining intensity (b) RT-PCR assays were performed to analyze the expression of the adipocyte marker genes in 3T3-L1-shLuc and -shAjuba cells at days 6 and 8. (c and d) Overexpression of Ajuba in 3T3-L1 cells evidently accelerates adipocyte maturation and increases the expression of adipocyte marker genes. Experiments were repeated three times in triplicate

with increasing amount of Myc-Ajuba in $293 \mathrm{~T}$ cells, and performed co-IP assays with Flag antibody. The co-eluted proteins were detected by western blot assays with Flag, HA or Myc antibodies, respectively. Consistently, PPARy readily immunoprecipitated CBP and Ajuba (Figure 7a, lanes 2 and 3). Strikingly, increasing the amount of Ajuba resulted in PPARy2 to co-immuoprecipitate more Ajuba and CBP (Figure 7a, lanes 3-6), suggesting Ajuba may facilitate the assembly of the PPAR $y$, Ajuba and p300/CBP ternary complex.

To examine the effect of PPARy, Ajuba and p300/CBP ternary complex in transactivating PPARy target promoter activity, we performed luciferase reporter assays on LPL promoter in 3T3-L1 cells. Consistently, PPARy2, Ajuba, p300 or CBP alone stimulated weak promoter activity; combination of PPARy2 and Ajuba, or PPARy2 and p300/CBP, or Ajuba and p300/CBP modestly induced the LPL promoter activity; whereas combination of PPARy2, Ajuba and p300/CBP stimulated the strongest LPL promoter activity (Figure $7 \mathrm{~b}$ ), suggesting a synergy between PPAR $\gamma$, Ajuba and p300/CBP exists at the PPARy target chromatins to activate gene expression.

To further examine the assembly of PPARy, Ajuba and p300/CBP ternary complex at endogenous chromatins, we performed ChIP assays in 3T3-L1-vector and 3T3-L1-Ajuba cells using antibodies specific to PPARy, Ajuba, p300, CBP and $\mathrm{H} 3-\mathrm{Ac}$, respectively. The enriched DNA fragments were amplified by $\mathrm{qPCR}$ using primer sets flanking the functional 

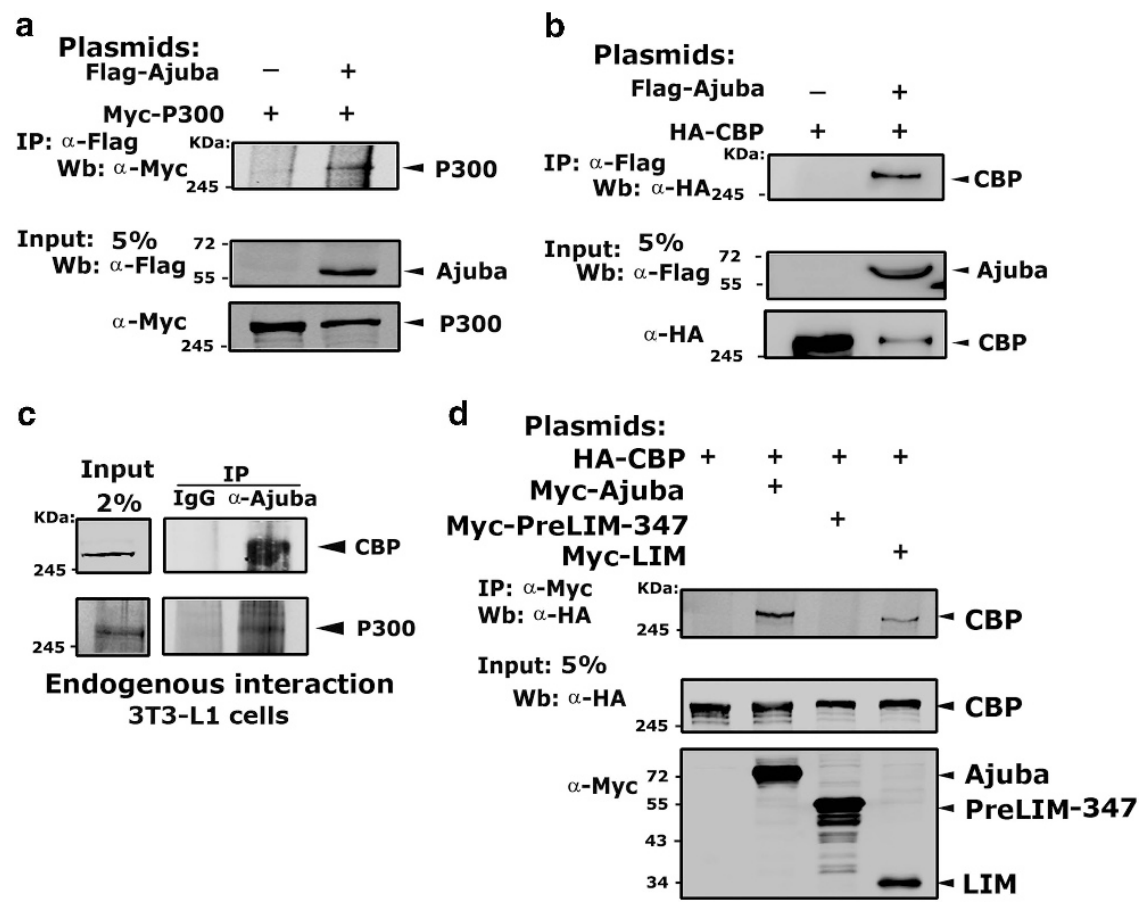

Figure 6 Ajuba interacts with p300/CBP. (a and b) Ajuba interacted with both p300 and CBP when co-expressed in 293 T cells. (c) Endogenous Ajuba readily co-immunoprecipitated p300 and CBP in 3T3-L1 cells. (d) The LIM domain interacts with p300/CBP. HA-CBP and full-length Myc-Ajuba, Myc-preLIM-347 or Myc-LIM were co-expressed in $293 \mathrm{~T}$ cells and co-IP assays were performed with Myc antibody

PPRE sites of LPL promoters. In 3T3-L1-vector cells, PPARy, Ajuba, p300 and CBP were found to be associated with PPARy target promoter at low level, and the histone acetylation remains relatively at low level represented by $\mathrm{H} 3-\mathrm{Ac}$ (Figure 7c). Consistently, stable overexpression of Ajuba in 3T3-L1 cells resulted in markedly increased binding of Ajuba, p300, CBP at the target chromatin and concomitantly markedly increased histone acetylation at this locus (Figure 7c). Collectively, these data demonstrate that Ajuba is required for PPARy to efficiently recruit p300/CBP to its target chromatins (Figure 7d).

Ajuba does not affect PPARy/RXR heterodimer formation. We previously demonstrated that Ajuba preferentially interacts with RXRY (22). To determine if Ajuba affects the PPARy/ $\mathrm{RXR}$ heterodimerization, we expressed Flag-PPARy2 and $\mathrm{HA}-\mathrm{RXR} a, \mathrm{RXR} y$ or HA-CBP, and together with increasing amount of Myc-Ajuba in 293 T cells. The Co-IP assays were carried out by using $\mathrm{HA}$ antibody. The co-eluted proteins were detected by western blot assays. Consistent to the results observed in Figure 7a, Ajuba increased the binding between PPARy2 and CBP (Supplementary Figure 2A, lanes 10-12); however, we did not observe any apparent change of the interaction between PPAR 2 and RXRa (Supplementary Figure 2A, lanes 2-5) or RXRy (Supplementary Figure 2A, lanes 6-9). To further strengthen these observations, we expressed Flag-PPARY2, Myc-Ajuba alone or in combination in $293 \mathrm{~T}$ cells, and performed co-IP assays using Flag antibody. The co-eluted Ajuba and endogenous CBP, RXRa and $\mathrm{RXR} y$ proteins were detected by western blot assays. Consistently, forced expression of Ajuba increased the interaction between PPARY2 and endogenous CBP protein, but had no effect on the interaction between PPARy2 and endogenous RXRa or RXRy (Supplementary Figure 2B). Collectively, these data indicate that although Ajuba can interact with both PPARy and RXR, it does not affect the PPARy/RXR heterodimer formation.

\section{Discussion}

Obesity is becoming an epidemic disease and is the major inductive factor for many metabolic disorders. Adipose tissue is the largest energy storage reservoir and has critical role in controlling the whole-body energy balance. ${ }^{4,36-37}$ Adipose tissue also functions as important endocrine organ and secretes numerous cytokines and hormones that regulate global energy homeostasis. ${ }^{38}$ In human, adipogenesis is mainly occurred during the process of embryo development and the turnover of adipocytes in adult adipose tissues. ${ }^{39,40}$ Thus, deciphering the adipocyte differentiation program will lead to eventual therapeutics to cure obesity-related metabolic diseases. In this report, we demonstrate Ajuba functions as an obligate co-activator of PPARy and is an important modulator for adipocyte differentiation. Ajuba binds the DNA-binding domain of PPAR $y$ in a ligand-independent manner and recruits p300/CBP via its LIM domain to activate PPARy target gene expression. These data suggest that Ajuba could be a potential therapeutic target for PPARy-related metabolic diseases.

Ajuba contains both nuclear localization and nuclear exporting signals and has the capacity of shuttling between the cytoplasm and nucleus. The subcellular localization of Ajuba at steady state varies in different cells and we showed that Ajuba can be found in both the cytoplasm and nucleus in 


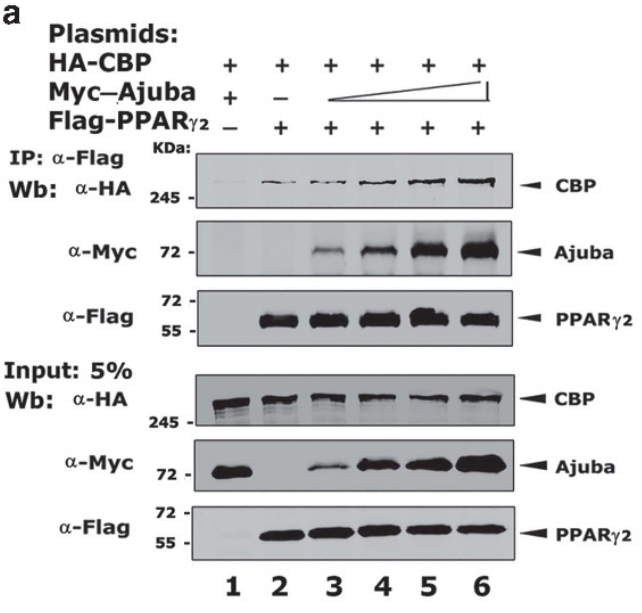

b

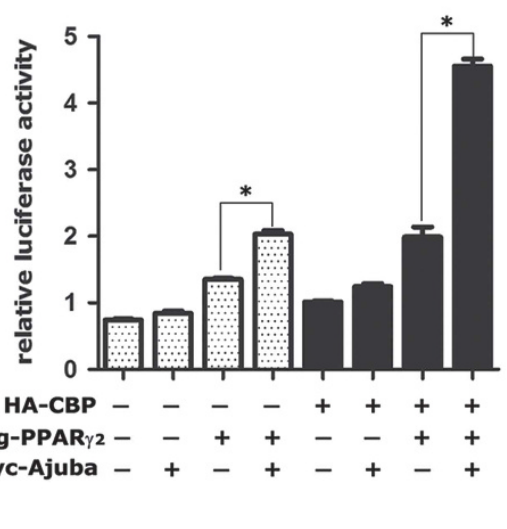

C

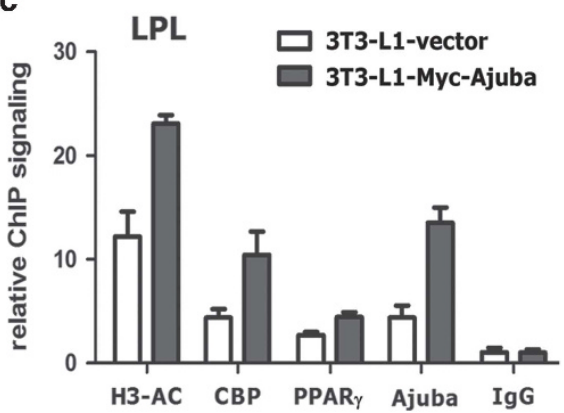

d

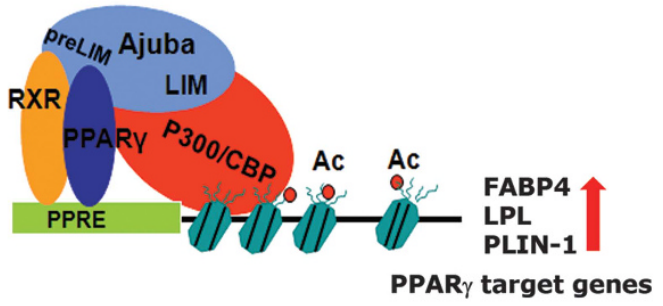

Figure 7 Ajuba recruits p300/CBP to PPAR $\gamma$ and its target chromatins. (a) PPAR $\gamma$, Ajuba and p300/CBP form a functional ternary complex. Flag-PPAR 2 and HA-CBP, together with increasing amount of Myc-Ajuba were co-expressed in $293 \mathrm{~T}$ cells, and co-IP assays were performed with Flag antibody. The co-eluted proteins were detected by western blot assays with Flag, HA or Myc antibodies, respectively. (b) Combination of PPAR $\gamma$, Ajuba and p300/CBP cooperatively stimulates LPL promoter activity. Luciferase reporter assays were performed in 3T3-L1 cells. The value was normalized to $\beta$-gal. Data were shown as mean \pm S.D. from three independent experiments, ${ }^{*} P<0.05$. (c) Ajuba is required for assembly of PPAR $\gamma$, Ajuba and p300/CBP ternary complex at endogenous chromatins. ChIP assays were performed in 3T3-L1-vector and 3T3-L1-Ajuba cells using antibodies specific to PPAR $\gamma$, Ajuba, p300/CBP or H3-Ac, respectively. The enriched DNA fragments were amplified by qPCR assays. Data: mean \pm S.D. from three independent experiments. (d) Model shows the PPAR $\gamma$, Ajuba and p300/CBP form a functional ternary complex to activate gene transcription

3T3-L1 cells. In the nucleus, Ajuba functions as a co-repressor for various transcription factors such as Gfi-1, Snail and ISI1 by recruiting HDACs and PRMT5. ${ }^{17,18,20,21,41}$ We recently discovered that Ajuba contains three conserved functional nuclear receptor (NR) boxes mediating a direct interaction with RAR $a$ and functions as a typical co-repressor for RAR $a$ in a ligand-dependent manner. ${ }^{22}$ Here, we demonstrate that Ajuba interacts with PPAR $y$ by different mechanism: the conserved NR boxes of Ajuba do not contribute to the interaction between Ajuba and PPAR $\gamma$, instead, a region in the preLIM is required for the interaction with PPARy; Ajuba binds the DNA-binding domain of PPARy in a ligand-independent manner. These findings suggest that PPARy and Ajuba may contain unique modules to mediate their specific interaction and protein complex assembly. The binding activities of Ajuba to nuclear receptors are reminiscence of the co-activator of PGC-1, which interacts with PPARa via its NR boxes, ${ }^{42}$ but interaction with PPAR $y$ is independent of these NR boxes. ${ }^{11,12}$ To better understand the interaction between Ajuba and nuclear receptors, a more detailed characterization of the binding motifs from these molecules will be necessary.

It is known that $\mathrm{p300/CBP}$ proteins are the major enzymes in the PPARy complexes to activate gene transcription. P300/
CBP can directly interact with AF1 and AF2 domains of PPARY or can be recruited by cofactors such as members of SRC family and PGC-1 family. 5,6,43,44 Here we showed that Ajuba constitutively recruits $\mathrm{p} 300 / \mathrm{CBP}$ to PPARy to transactivate gene expression independent of ligand stimulation. Whether Ajuba is a component of the known PPAR $/$ SRC/p300/CBP complex is not clear, but a comprehensive proteomic analysis of the Ajuba-associated protein complexes will help to understand the Ajuba/PPARy cooperativeness. In addition to modulate PPARy signaling, Ajuba may regulate other signaling molecules involved in the adipocyte differentiation program. We are currently employing multiple approaches including proteomics, ChIP-seq, RNA-seq and Ajuba-null mouse model to explore the role of Ajuba in the regulation of adipogenesis.

\section{Materials and Methods}

Plasmids. Myc or Flag-tagged Ajuba full-length and its truncation mutants were previously described. ${ }^{20}$ The murine Ajuba cDNA was subcloned into pBabe-puro vector and the Myc-tag was added at the N-terminus of Ajuba protein. We used the pBabe-Ajuba plasmid as a template and subcloned the Ajuba-coding DNA sequence into $\mathrm{pET} 28 \mathrm{a}$-vector and got the $\mathrm{pET} 28 \mathrm{a}$-Ajuba plasmid with the His tag at the N-terminus of Ajuba. The plasmids of PLKO.1-shAjuba and PLKO.1-shLuc were described previously.22 The plasmids of Myc-tagged Limd1, WTIP, LPP and Zyxin 
have been described previously. ${ }^{17}$ The plasmids of pCDNA3.1-Flag-PPAR $\gamma 1$, Flag- PPAR $\gamma 2$ and its truncation mutants were cloned from murine PPAR $\gamma 1$ and PPAR $\gamma 2$ cDNA. The plasmid pGEX-4T-1-PPAR $\gamma-C$ was subcloned from pCDNA3.1-Flag-PPAR $\gamma 2$ plasmids. HA-CBP and Myc-p300 were kindly gifted by Dr. Eugene $Y$ Chin at the Institute of Health Sciences of Chinese Academy of Sciences. The human RXR $\alpha / \gamma$ cDNA was subcloned into pCDNA3.0-6HA-vector by PCR method. The murine LPL gene promoter from -445 to +73 containing a functional PPRE site was amplified by PCR and subcloned into pGL3.0-Luc basic vector between Kpnl and Xhol sites to create the pGL3-mLPL-Luc reporter.

Cell culture, transfection, retroviral infection and luciferase reporter assay. 293 T and 3T3-L1 (ATCC, Manassas, VA, USA) were cultured in Dulbecco's modified Eagle's medium supplemented with $10 \%$ FBS, $2 \mathrm{mML}$ glutamine and penicillin $(50 \mathrm{U} / \mathrm{ml}) /$ streptomycin $(50 \mu \mathrm{g} / \mathrm{ml})$ at $37^{\circ} \mathrm{C}$ under $5 \% \mathrm{CO}_{2}$ in a humidified chamber. The transfection was performed using Lipofectamine 2000 (Invitrogen, Carlsbad, CA, USA) as described. ${ }^{22}$ Supernatants containing viruses were packed from $293 \mathrm{~T}$ cells. When growing to $40-60 \%$ confluence, 3T3-L1 cells were infected with viral supernatants, and $5 \mu \mathrm{g} / \mathrm{ml}$ puromycin was added to select the stable cells. The luciferase reporter assays were described previously. ${ }^{22}$

Co-IP, GST pull-down, western blot, IF and antibodies. IP, IF and western blotting assays were carried out as described. ${ }^{20,22}$ The antibodies used are as follows: anti-Myc (13-2500, Invitrogen), anti-Flag (F3165, F7425, Sigma, St. Louis, MO, USA), anti-HA (MMS-101P, Covance, Emeryville, CA, USA), antiPPAR (sc-7273, Santa Cruz, Dallas, TX, USA), anti-CBP (A-22 Santa Cruz), antip300 (N-15, sc-584, Santa Cruz), anti-RXR $\alpha$ (D20,sc-553, Santa Cruz), anti-RXR $\gamma$ (Y20,SC-555, Santa Cruz), anti-Acetyl-H3 (06-559, sc-369, Millipore, Temecula, CA, USA) and anti- $\beta$-actin (60008-1-lg, Proteintech, Chicago, IL, USA), normal mouse IgG (sc-2025, Santa Cruz), normal rabbit IgG (sc-2027, Santa Cruz). The rabbit Ajuba antibody was described previously. ${ }^{22}$ pGEX-4 T-1-PPAR $\gamma$-C and PET28aAjuba were expressed in BL21 E. coli, respectively, and the pull-down assays were performed by using GST beads (17-0756-01, GE Healthcare, Uppsala, Sweden) and Ni-beads(17-5318-06, GE Healthcare).

RT-PCR and qPCR. Exponentially growing $3 T 3-\mathrm{L} 1$ cells in 10-cm dish were treated with $1 \mu \mathrm{M}$ Rosi or corresponding amount of DMSO for $16 \mathrm{~h}$, and then the resulting cells were harvested for RNA isolation using the Trizol reagent (Ambion, Carlsbad, CA, USA). The reverse transcription procedure was described previously. ${ }^{22}$ Semi-quantitative PCR or qPCR assays were carried out to examine the level of PPAR $\gamma$ target genes expression. The level of 36B4 or GAPDH was used as the endogenous control. The qPCR assays were performed with SYBR reagent (ABI 7500 Fast).

Chromatin immunoprecipatation. ChIP assays were carried out in 3T3-L1 cells and 293 T cells. The procedure has been decribed previously. ${ }^{22}$ The chromatin was sonicated to fragments ranging from 500 to $800 \mathrm{bp}$ in size. Immunoprecipitation was performed using antibodies against Flag-tag (mouse), Myc-tag (mouse), Ajuba, PPAR $\gamma$, CBP, p300 and Acetyl-H3. Equal amount of rabbit normal IgG was used as control. The precipitated DNA fragments were examined by $q P C R$.

3T3-L1 cells differentiation and Oil-Red-O staining. The 3T3-L1 cells were grown to confluency for 2 days and then were cultured in induction media containing the cocktail of $10 \mu \mathrm{g} / \mathrm{ml}$ insulin (I5500, Sigma), $1 \mu \mathrm{M}$ dexamethasone (D4902, Sigma), 0.5 mM IBMX (17018, Sigma), with $2.5 \mu$ M Rosi (R2408, Sigma) or not for 2 days. Adding Rosi in the media significantly enhances adipogenic differentiation. The day adding the induction media was designated as day 0 . At day 3 , fresh media containing $10 \mu \mathrm{g} / \mathrm{ml}$ insulin were added for another 2 days. Further differentiation is allowed in normal culture media. At designated time, cells were fixed in $4 \%$ paraformaldehyde and the adipocytes were visualized by Oil-Red-0 staining. For each time point, triplicate wells were set for each condition and two wells were fixed and staining. Four images were randomly taken for each well using phase contrast microscopy under $\times 200$. The differentiation degree was quantified by the averaged red-staining intensity (after Oil-Red-O staining) by using Photoshop software. The remaining wells were extracted for RNA isolation and qRT-PCR.

\section{Conflict of Interest}

The authors declare no conflict of interest.
Acknowledgements. We thank Dr. Eugene Y Chin for the gift of the p300 and CBP plasmids. This work was supported by the Ministry of Sciences and Technology of China (2013CB 910900); the National Science Foundation of China (No.81172028, 81372309); Shanghai Committee of Science and Technology (13JC1401302), Shanghai Municipal Commission of Health and Family Planning (No. 201440426), and Science and Technology Commission of Changning District of Shanghai No. CNKW2014F01).

1. Tang QQ, Lane MD. Adipogenesis: from stem cell to adipocyte. Annu Rev Biochem 2012; 81 715-736.

2. Rosen ED, MacDougald OA. Adipocyte differentiation from the inside out. Nat Rev Mol Cell Biol 2006; 7: 885-896.

3. Cristancho AG, Lazar MA. Forming functional fat: a growing understanding of adipocyte differentiation. Nat Rev Mol Cell Biol 2011; 12: 722-734.

4. Rosen Evan D, Spiegelman Bruce M. What We Talk About When We Talk About Fat. Cell 2014: 156: 20-44.

5. Ahmadian M, Suh JM, Hah N, Liddle C, Atkins AR, Downes M et al. PPAR $\gamma$ signaling and metabolism: the good, the bad and the future. Nat Med 2013; 19: 557-566.

6. Lefterova MI, Haakonsson AK, Lazar MA, Mandrup S. PPARgamma and the global map of adipogenesis and beyond. Trends Endocrinol Metab 2014; 25: 293-302.

7. Barak Y, Nelson MC, Ong ES, Jones YZ, Ruiz-Lozano P, Chien KR et al. PPAR gamma is required for placental, cardiac, and adipose tissue development. Mol Cell. 1999; 4: 585-595.

8. Ren D, Collingwood TN, Rebar EJ, Wolffe AP, Camp HS. PPARgamma knockdown by engineered transcription factors: exogenous PPARgamma2 but not PPARgamma1 reactivates adipogenesis. Genes Dev 2002; 16: 27-32.

9. Gelman L, Zhou G, Fajas L, Raspé E, Fruchart JC, Auwerx J. p300 interacts with the N- and C-terminal part of PPARgamma2 in a ligand-independent and -dependent manner, respectively. J Biol Chem 1999; 274: 7681-7688.

10. Castillo G, Brun RP, Rosenfield JK, Hauser S, Park CW, Troy AE et al. An adipogenic cofactor bound by the differentiation domain of PPARgamma. EMBO J 1999; 18: 3676-3687.

11. Lin J, Handschin C, Spiegelman BM. Metabolic control through the PGC-1 family of transcription coactivators. Cell Metab 2005; 1: 361-370.

12. Puigserver $P, W u Z$, Park CW, Graves R, Wright M, Spiegelman BM. A cold-inducible coactivator of nuclear receptors linked to adaptive thermogenesis. Cell 1998; 92: 829-839.

13. Lonard DM, O'Malley BW. Nuclear receptor coregulators: modulators of pathology and therapeutic targets. Nat Rev Endocrinol 2012; 8: 598-604.

14. Schmeichel KL, Beckerle MC. The LIM domain is a modular protein-binding interface. Cell 1994; 79: 211-219.

15. Kadrmas JL, Beckerle MC. The LIM domain: from the cytoskeleton to the nucleus. Nat Rev Mol Cell Biol 2004; 5: 920-931.

16. Goyal RK, Lin P, Kanungo J, Payne AS, Muslin AJ, Longmore GD. Ajuba, a novel LIM protein, interacts with Grb2, augments mitogen-activated protein kinase activity in fibroblasts, and promotes meiotic maturation of Xenopus oocytes in a Grb2- and Ras-dependent manner. Mol Cell Biol 1999; 19: 4379-4389.

17. Langer EM, Feng $Y$ et al. Ajuba LIM proteins are snail/slug corepressors required for neural crest development in Xenopus. Dev Cell 2008; 14: 424-436.

18. Ayyanathan K, Peng H, Hou Z, Fredericks WJ, Goyal RK, Langer EM et al. The Ajuba LIM domain protein is a corepressor for SNAG domain mediated repression and participates in nucleocytoplasmic Shuttling. Cancer Res 2007; 67: 9097-9106.

19. Kanungo J, Pratt SJ, Marie H, Longmore GD. Ajuba, a cytosolic LIM protein, shuttles into the nucleus and affects embryonal cell proliferation and fate decisions. Mol Biol Cell 2000; 11: 3299-3313.

20. Hou Z, Peng H, Ayyanathan K, Yan KP, Langer EM, Longmore GD et al. The LIM protein AJUBA recruits protein arginine methyltransferase 5 to mediate SNAIL-dependent transcriptional repression. Mol Cell Biol 2008; 28: 3198-3207.

21. Montoya-Durango DE, Velu CS, Kazanjian A, Rojas ME, Jay CM, Longmore GD et al. Ajuba functions as a histone deacetylase-dependent co-repressor for autoregulation of the growth factor-independent-1 transcription factor. J Biol Chem 2008; 283: 32056-32065.

22. Hou Z, Peng H, White DE, Negorev DG, Maul GG, Feng Y et al. LIM protein Ajuba functions as a nuclear receptor corepressor and negatively regulates retinoic acid signaling. Proc Natl Acad Sci USA 2010; 107: 2938-2943.

23. Marie H, Pratt SJ, Betson M, Epple H, Kittler JT, Meek L et al. The LIM protein Ajuba is recruited to cadherin-dependent cell junctions through an association with alpha-catenin. J Biol Chem 2003; 278: 1220-1228.

24. Pratt SJ, Epple H, Ward M, Feng Y, Braga VM, Longmore GD. The LIM protein Ajuba influences p130Cas localization and Rac1 activity during cell migration. J Cell Biol 2005; 168 : 813-824.

25. Kisseleva M, Feng Y, Ward M, Song C, Anderson RA, Longmore GD. The LIM protein Ajuba regulates phosphatidylinositol 4,5-bisphosphate levels in migrating cells through an interaction with and activation of PIPKI alpha. Mol Cell Biol 2005; 25 : 3956-3966. 
26. Nola S, Daigaku R, Smolarczyk K, Carstens M, Martin-Martin B, Longmore G et al. Ajuba is required for Rac activation and maintenance of E-cadherin adhesion. J Cell Biol 2011; 195 : 855-871.

27. Hirota T, Kunitoku N, Sasayama T, Marumoto T, Zhang D, Nitta M et al. Aurora-A and an interacting activator, the LIM protein Ajuba, are required for mitotic commitment in human cells. Cell 2003; 114: 585-598.

28. Feng Y, Longmore GD. The LIM protein Ajuba influences interleukin-1-induced NF-kappaB activation by affecting the assembly and activity of the protein kinase Czeta/p62/TRAF6 signaling complex. Mol Cell Biol 2005; 25: 4010-4022.

29. James V, Zhang Y, Foxler DE, de Moor CH, Kong YW, Webb TM. LIM-domain proteins, LIMD1, Ajuba, and WTIP are required for microRNA-mediated gene silencing. Proc Natl Acad Sci USA 2010; 107: 12499-12504.

30. Das Thakur M, Feng Y, Jagannathan R, Seppa MJ, Skeath JB, Longmore GD. Ajuba LIM proteins are negative regulators of the Hippo signaling pathway. Curr Biol 2010; 20: 657-662.

31. Hou Z, Peng H, White DE, Wang P, Lieberman PM, Halazonetis T et al. 14-3-3 binding sites in the snail protein are essential for snail-mediated transcriptional repression and epithelialmesenchymal differentiation. Cancer Res 2010; 70: 4385-4393.

32. Tontonoz P, Hu E, Graves RA, Budavari Al, Spiegelman BM. mPPAR gamma 2: tissuespecific regulator of an adipocyte enhancer. Genes Dev 1994; 8: 1224-1234.

33. Teboul L, Febbraio M, Gaillard D, Amri EZ, Silverstein R, Grimaldi PA. Structural and functional characterization of the mouse fatty acid translocase promoter: activation during adipose differentiation. Biochem J 2001; 360: 305-312.

34. Schoonjans K, Peinado-Onsurbe J, Lefebvre AM, Heyman RA, Briggs M, Deeb S et al. PPARalpha and PPARgamma activators direct a distinct tissue-specific transcriptional response via a PPRE in the lipoprotein lipase gene. EMBO J 1996; 15: 5336-5348.
35. Nagai S, Shimizu C, Umetsu M, Taniguchi S, Endo M, Miyoshi H et al. Identification of a functional peroxisome proliferator-activated receptor responsive element within the murine perilipin gene. Endocrinology 2004; 145: 2346-2356.

36. Rosen ED, Spiegelman BM. Adipocytes as regulators of energy balance and glucose homeostasis. Nature 2006; 444: 847-853.

37. Hamdy O, Porramatikul S, Al-Ozairi E. Metabolic obesity: the paradox between visceral and subcutaneous fat. Curr Diabetes Rev 2006; 2: 367-373.

38. Galic S, Oakhill JS, Steinberg GR. Adipose tissue as an endocrine organ. Mol Cell Endocrinol 2010; 316: 129-139.

39. Spalding KL, Arner E, Westermark PO, Bernard S, Buchholz BA, Bergmann O et al. Dynamics of fat cell turnover in humans. Nature 2008; 453: 783-787.

40. Arner P, Spalding KL. Fat cell turnover in humans. Biochem Biophys Res Commun 2010; 396: 101-104.

41. Witzel HR, Jungblut B, Choe CP, Crump JG, Braun T, Dobreva G. The LIM protein Ajuba restricts the second heart field progenitor pool by regulating IsI1 activity. Dev Cell 2012; 23: 58-70.

42. Vega RB, Huss JM, Kelly DP. The coactivator PGC-1 cooperates with peroxisome proliferator-activated receptor alpha in transcriptional control of nuclear genes encoding mitochondrial fatty acid oxidation enzymes. Mol Cell Biol 2000; 20: 1868-1876.

43. Gelman L, Zhou G, Fajas L, Raspe E, Fruchart JC, Auwerx J. p300 interacts with the N- and C-terminal part of PPARgamma2 in a ligand-independent and -dependent manner, respectively. J Biol Chem 1999; 274: 7681-7688.

44. Wallberg AE, Yamamura S, Malik S, Spiegelman BM, Roeder RG. Coordination of p300-mediated chromatin remodeling and TRAP/mediator function through coactivator PGC-1alpha. Mol Cell 2003; 12: 1137-1149.

\section{Supplementary Information accompanies this paper on Cell Death and Differentiation website (http://www.nature.com/cdd)}

